\title{
Crime Investigation and Criminal Network Analysis Using Archive Call Detail Records
}

\author{
Dr. Manish Kumar ${ }^{1}$ \\ Asst. Professor, Dept. of Computer Applications, \\ M. S. Ramaiah Institute of Technology, \\ Bangalore-560 054, INDIA \\ E-Mail:- manishkumarjsr@yahoo.com
}

\author{
Dr. M. Hanumanthappa ${ }^{2}$ \\ Professor, Dept. of Computer Science and Applications, \\ Jnana Bharathi Campus, Bangalore University, \\ Bangalore -560 056, INDIA
}

\author{
Dr. T. V. Suresh Kumar ${ }^{3}$ \\ Professor \& Head, Dept. of Computer Applications, \\ M. S. Ramaiah Institute of Technology, Bangalore-560 054, INDIA
}

\begin{abstract}
Mobile phones are widely used in our day-to-day life. It's not only used by a common man but also used by antisocial elements and that's why it's not a surprise that today in almost every case the first step towards solving a Crime is to analyze the Call Records of the Suspects. Today in almost all the criminal cases, analysis of Mobile Phone calls of suspect's plays an important role in investigation of crime. In order to track the suspects/criminal, investigative agency need to analyze Call Detail Record (CDR) of the suspect's received in varied formats from the service providers. It is generally found that the anti-social elements have their own network and association with other criminals and anti-social elements. Many time they are associated together for committing a crime or they may be knowing about each other activities and crimes. Many of their associates might have been convicted or their name might have been recorded in old cases. Archiving the CDR in centralize database benefit the investigative agency for identification of possible suspects in other cases. This research paper discuss the implementation model for investigating the case using achieve CDR.
\end{abstract}

Keywords-Call Detail Record (CDR), International Mobile Equipment Identity (IMEI), Mobile Service Provider, Crime Investigation, Mobile Phones, Crime Data Analysis.

\section{Introduction}

The objective of crime data analysis is to identify the structure and patterns which exist among the criminals and anti-social element. Such analysis will help to solve many unsolved cases and can also give a crucial information to the investigative agency about the association among the criminals.

Police departments typically maintain their own database in which crime details, arrests, geo location of crime and many other relevant information related to the crimes are stored. Even though these systems are slightly different from agency to agency, the basic purposes and functions are the same.
These systems are key systems for data in policing and crime analysis.

Historically and still today, it is a great struggle for investigative agency and crime analysts to retrieve data from their agencies' systems. In many cases, customized software create functions for producing paper reports and retrieving one case at a time. In general these customized software do not give options for downloading large amounts of data needed for analysis purposes.

There are various sources of data which investigation agency generally use for investigating the case. One among them is Call Detail Record (CDR). A call detail record (CDR) is a data record produced by a telephone exchange or other telecommunications equipment that documents the details of a telephone call or other telecommunications transaction (e.g., text message) that passes through that facility or device[2][3].

In this paper we are discussing a model to show how a Call Detail Record (CDR) Database which contain the CDR related to old cases and convicted criminals can be used for investigating the case.

\section{Problem Defination and Objectives}

It's not a surprise that today in almost every case the First step towards solving a Crime is to analyze the Call Records of the Suspects. This often takes a lot of time and effort. The time and complexity of analysis increases based on the volume of CDR collected.

CDR analysis become a vital part of all crime investigation. However it is observed that in most of the cases investigative agency normally collect the CDR in context to the case and specific suspects. Most of the time they analyze it keeping in view of particular case and involvement of 
individual suspect with the case. Once the case is solved, these CDRs are thrown away.

It is also observed that analyzing a CDR is really very tough and tedious task, and hence a software tool is require. There are plenty of such tools available in market but as mentioned earlier, it can help to analyze the CDR in context of specific case and suspects.

We are proposing that the CDR related to the old cases and convicted criminals can be preserved in a centralized database, which may be called as CDR Database, for the purpose of analysis and investigation purpose in other cases. Analysis based on CDR Database can be useful to identify the association of criminals or like-minded, involved in many other cases.

\section{Proposed Model}

In this paper we are proposing a graph based analytical model using the CDR database. The CDR database contains the CDR of old cases and the criminals associated in those crimes. Our objective is to build a model which can assist the investigative agency for investigating the cases with the help of CDR.

It is generally found that the anti-social elements have their own network and association with other criminals and antisocial elements. Many time they are associated together for committing a crime or they may be knowing about each other activities and crimes. Many of their associates might have been convicted or their name might have been recorded in old cases. If the CDR of old cases and the suspects are stored in centralized database, it can be useful for investigation.

Analyzing these association among the anti-social elements can help the investigative agency to crack many cases. However, identifying caller numbers and their relationships in tabular data is very hard. We are thus using a graphical representation, which is based on graph theory. This graph can pictorially show how the phone numbers/IMEI are connected by other phone calls/IMEI number.

\section{Implementation Illustration of Proposed Model}

This section describe our CDR analysis model. For illustration purpose we have simplified the whole process and shown CDR table in very simple form, whereas practically it contains many fields and attributes which is important for investigation. Some of the most important attributes in CDR are Caller Number, IMEI number of Caller, Called Number, and Location from where call is generated etc. [1][4]. For illustration purpose we are showing only few fields in example.

It's important to note that IMEI number which is one of the vital field in CDR is not shown in the explanation to keep the illustration simple. IMEI number is a unique number for each mobile and it's important for investigation because even if the suspects changes his/her service provider or mobile number, the IMEI number remain same unless and until he/she change the equipment (mobile phone). The next section discuss the working model in details with simplified example.

\section{A. Call Detail Record Analysis}

Today in most of the crime investigation, Call Record Data analysis play a vital role. Investigative agency collects the Call detail record of suspects from the mobile service provider. A call detail record (CDR) is data produced by a telephone exchange or other telecommunications equipment (Figure 1) which contains the details of a telephone call or other telecommunications transaction (e.g., text message) that passes through that facility or device.

A call detail record contains metadata - that is, data about data - containing data fields that describe a specific instance of a telecommunication. It does not include the content of the communication. The record contains various attributes of the call, such as time, duration, completion status, source number, and destination number.

In general, a call detail record describing a particular

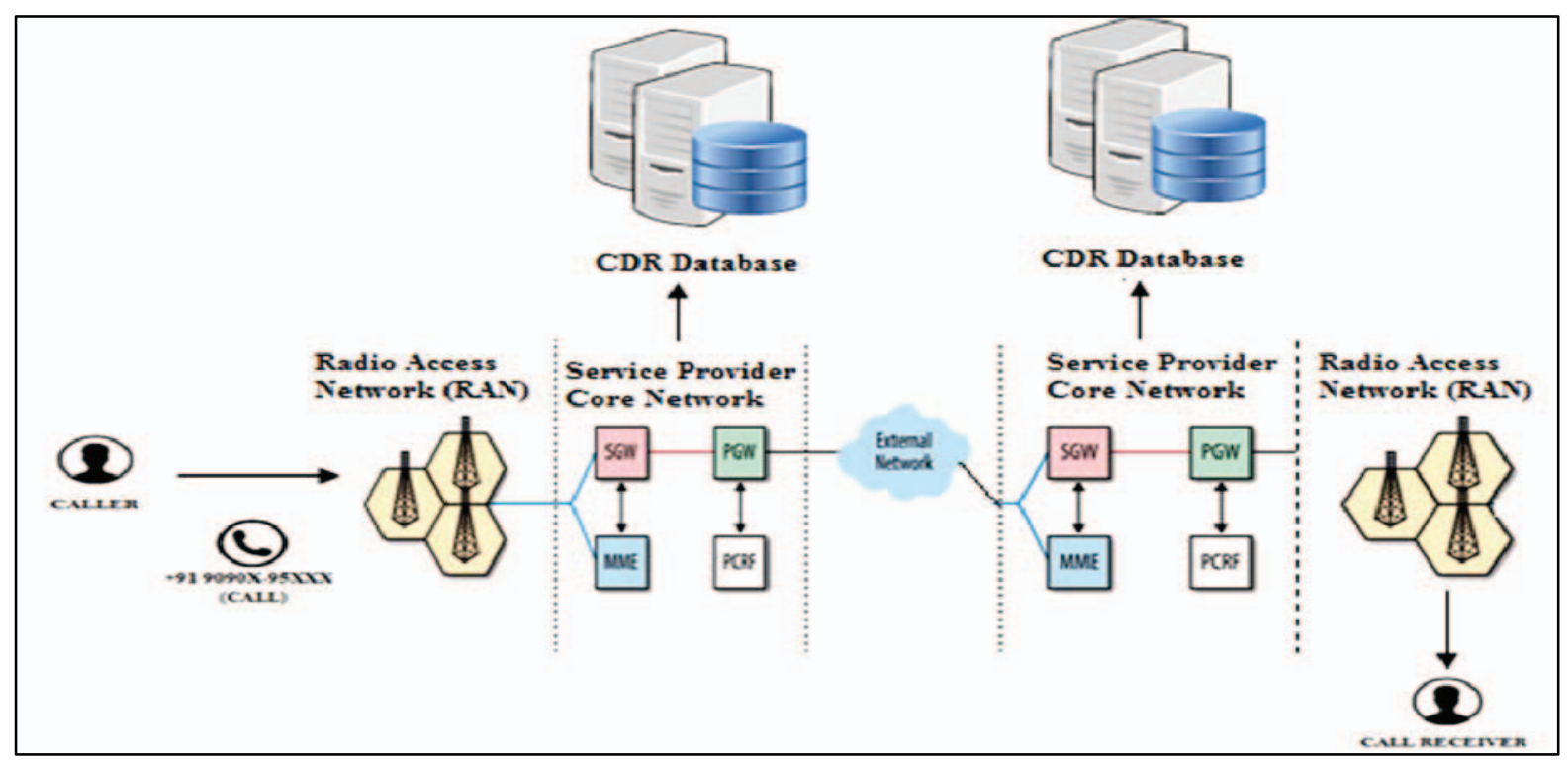

Fig. 1. Source of CDR in Telecommunication Network 


\section{IEEE Eighth International Conference on Advanced Computing (ICoAC)}

phone call might include the following attributes:

- Phone Number of the subscriber (Calling Person Number)

- Phone Number of the called person (Call Receiving Person).

- $\quad$ Starting time of the call (Date and Time)

- Call duration

- Billing phone number ( Number which is charged for the call)

- Unique Identification Number of the Telephone Exchange or Equipment (IMEI Number).

- A unique sequence number for identifying the record

- Disposition or the results of the call, indicating, for example, whether or not the call was connected

- $\quad$ Call type (Voice, SMS, etc.)

- Any fault condition encountered etc.

In actual modern practice, call detail records are much more detailed. It may contain many more attributes including tower geographical location details (Latitude, Longitude), cell ID, priority of services and many other details which may vary from service provider to service provider.
We propose that these CDR of individual case can be stored in a database for long term analysis. It is generally found that the anti-social elements have their own network and association with other criminals and anti-social elements. Many time they are associated together for committing a crime or they may be knowing about each other activities and crimes. Analyzing these association among the anti-social elements can help the investigative agency to crack many cases. However, identifying caller numbers and their relationships in tabular data is very hard. We are thus using a graphical representation, which is based on graph theory. The pictorial graph representation can show how the phone numbers are connected by other phone calls. To illustrate the whole process, in next section we are assuming one scenario. The name and number shown in the example are fictitious.

\section{B. Investigation Using Call Detail Records}

Assume that one robbery took place at 11th Cross, Malleswaram, Bangalore on 20th November 2015 at 10:30 PM. What we need first, is to identify the suspected criminals who made the phone calls from the crime location during a specific date and time of the crime.

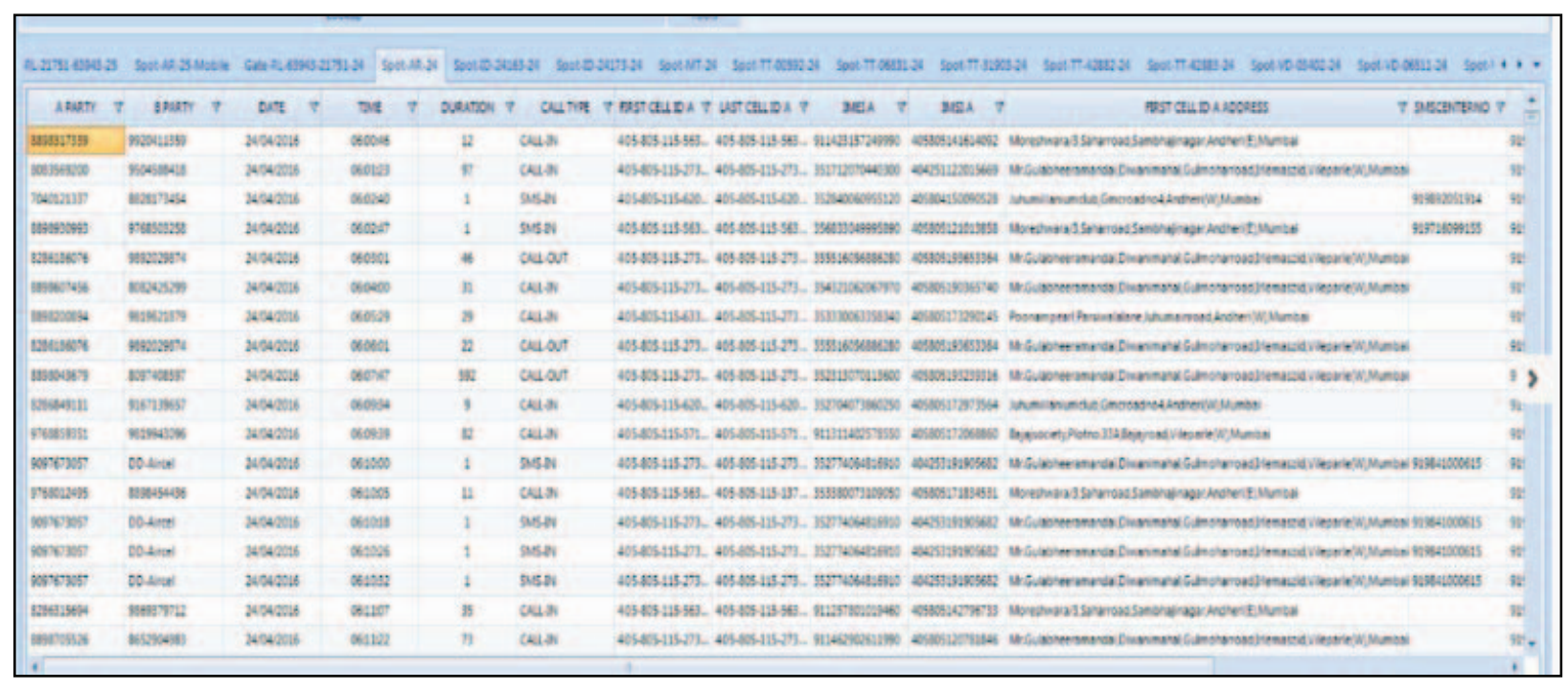

Fig. 2. Call Detail Record Sample Format

The Call Record Data provided by telecommunication service providers to the law enforcement agency is normally in standard tabular format (i.e. .xls or .csv file format as shown in Figure 2). These records are provided to the investigative agency on special request, after following some mandatory legal formalities. These file in tabular format sometime contains massive records and is challenging task for the investigator to analyze it and to make any interference [5]. Investigative agency takes the help of in-house customized tools to do the analysis of CDR. Most of the time they analyze it keeping in view of particular case and involvement of individual suspect with the case. Once the case is solved, these CDR is thrown away.

\section{Identifying The Potential Suspect}

In the above mentioned case, the police officers would ask the mobile service provider for the phone calls made 60 minutes before and after 10:30 PM near 11th Cross, Malleswaram, Bangalore on 20th November 2015. Here is how a service provider can quickly answer that question using simple query.

SELECT Caller_Number, Called_Number, Call_Start_Time, Call_Duration

Where Cell.Site='0101' OR Cell.Site='0102' OR Cell.Site= '0103' AND Call_Start_Time BETWEEN 21:30 AND 23:30; 
The query above looks for the phone calls made from 3 of the nearest towers (query can be generated for ' $n$ ' number of towers based on the number of tower serving in the area) from 11th Cross, Malleswaram, Bangalore, where the call started between 9:30 PM and 11:30 PM on 20th November 2015.

Let us assume that the query give us 5 records. Table 1 shows the results of the query. These five people may be potential suspects. They all have made a phone call in the vicinity of crime location. In real life situation these numbers may be in thousand and practically it is not possible to suspects all the caller as a potential criminal. So now the question is, among these, who may be a potential suspect and how to identify them?

TABLE I.

CAll Detail ReCORD OF CRIME SCENE

\begin{tabular}{|c|c|c|c|c|}
\hline $\begin{array}{c}\text { Sl. } \\
\text { No. }\end{array}$ & $\begin{array}{c}\text { Caller } \\
\text { Number }\end{array}$ & $\begin{array}{c}\text { Called } \\
\text { Number }\end{array}$ & Time & $\begin{array}{c}\text { Duration(in } \\
\text { Min) }\end{array}$ \\
\hline 1 & 99XX6796X1 & 99XX679X18 & $21: 45: 05$ & 08 \\
\hline 2 & 99XX6796X2 & 99XX679X19 & $22: 14: 45$ & 09 \\
\hline 3 & 99XX6796X3 & 99XX679X17 & $23: 11: 02$ & 05 \\
\hline 4 & 99XX6796X4 & 99XX679X16 & $23: 20: 00$ & 02 \\
\hline 4 & 99XX6796X5 & 99XX679X15 & $23: 25: 06$ & 03 \\
\hline 5 & 99XX6796X6 & 99XX679X14 & $23: 29: 02$ & 01 \\
\hline
\end{tabular}

\section{Identifying The Network of Suspects}

We need further analysis to identify our perpetrator. We can merge the above CDR (Table 1) with CDR database. The CDR database contains the CDR of old cases and associated criminals. Now we can analyze if there any relation exist between these callers with the other criminal involved in older cases. If we find any such caller, that can be the potential preparatory. Once the first level of analysis is done number of suspects can be reduced from thousand to hundreds.

Table 2 represents such scenario. The highlighted rows in table 2 represent the caller numbers, who has some association with the convicted criminal. Now we need to collect the CDR of the suspected perpetrator (last 30-50 days call record). These CDR can be merged with the CDR database for further analysis to find out if they have any relation with the criminals

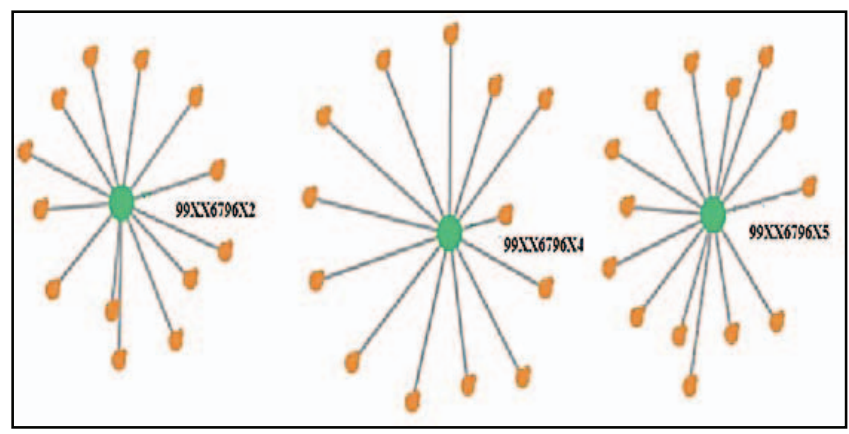

Fig. 5. The suspects and the calls they made

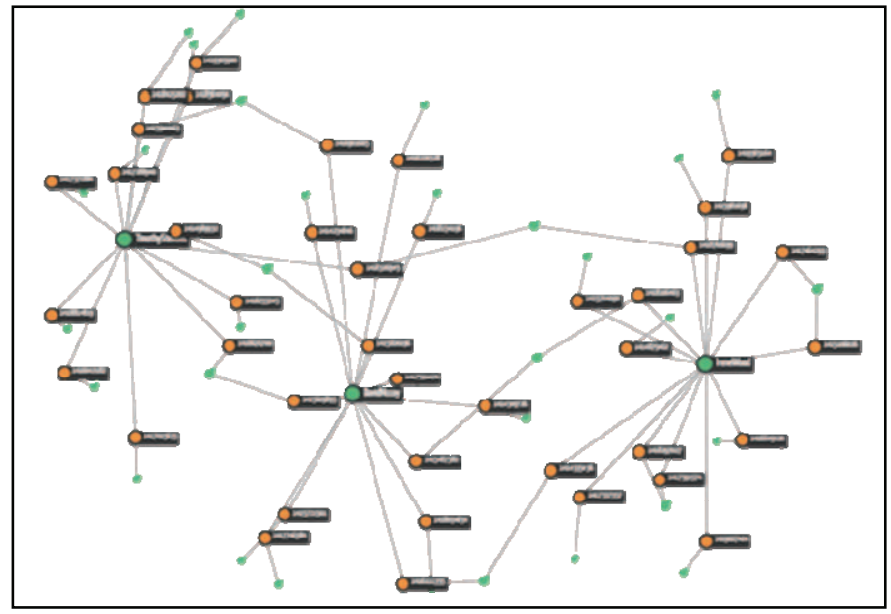

Fig. 3. The suspects, the calls they made and who they made it

involved in some of the old cases. These are the 2 nd degree contacts of our suspects. The graphical results of their connection may look like as shown in Figure 3:

TABle II. Suspected Perpetrators (Highlighted Rows)

\begin{tabular}{|c|c|c|c|c|}
\hline $\begin{array}{c}\text { Sl. } \\
\text { No. }\end{array}$ & Caller Number & Called Number & Time & $\begin{array}{c}\text { Duration(in } \\
\text { Min) }\end{array}$ \\
\hline 1 & 99XX6796X1 & 99XX679X18 & $21: 45: 05$ & 08 \\
\hline 2 & 99XX6796X2 & 99XX679X19 & $22: 14: 45$ & 09 \\
\hline 3 & 99XX6796X3 & 99XX679X17 & $23: 11: 02$ & 05 \\
\hline 4 & $\mathbf{9 9 X X 6 7 9 6 X 4}$ & $\mathbf{9 9 X X 6 7 9 X 1 6}$ & $23: 20: 00$ & 02 \\
4 & $\mathbf{9 9 X X 6 7 9 6 X 5}$ & $\mathbf{9 9 X X 6 7 9 X 1 5}$ & $23: 25: 06$ & 03 \\
\hline 5 & $\mathbf{9 9 X X 6 7 9 6 X 6}$ & $\mathbf{9 9 X X 6 7 9 X 1 4}$ & $23: 29: 02$ & 01 \\
\hline
\end{tabular}

Above we can see the phone calls made by each of our suspects. If we want to see the persons our suspects are in contact with, we have to display the persons connected to the calls. Figure 4 shows the visual representation of suspects call and their relation among the criminals involved in some of the recorded crime in police record. As you can visually identify that in Figure 4, nodes mention the names of the person. It is

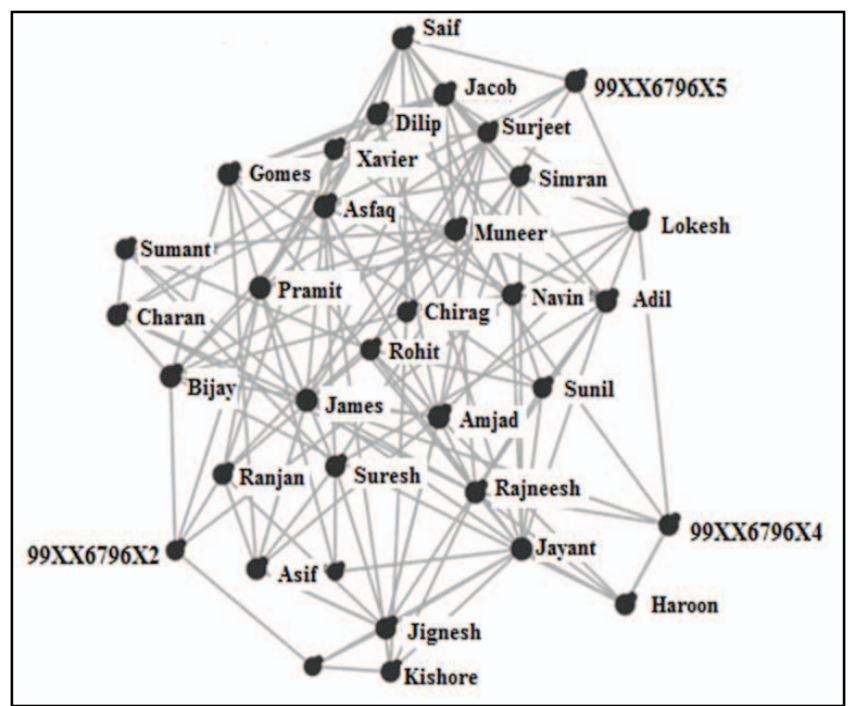

Fig. 4. The network of suspects with the convicted criminals 
possible because the police CDR database mention the name of the criminal, so it is possible to map the number vs. name. It gives more easiness for the investigator to analyze the relation. Graph visualization makes it easy to search and understand connected persons. The picture above sums up the network of our suspects. That information would have require a long investigation with Excel or with traditional analytical tools.

\section{v. Visual Analysis of The Suspects Network}

After identifying the suspects, next step is to do visual analysis of their network to zero down the suspect. Now in our example, let's see how the network of our 3 suspects, 99XX6796X2, 99XX6796X4 and 99XX6796X5. Figure 5 shows the network of our 3 suspects with the convicted criminal in police record. The graph is fairly dense and thus hard to read and analyze.From the above graph we can select any one of the suspects to see individual suspect connections with the convicted criminals as shown in Figure 6.

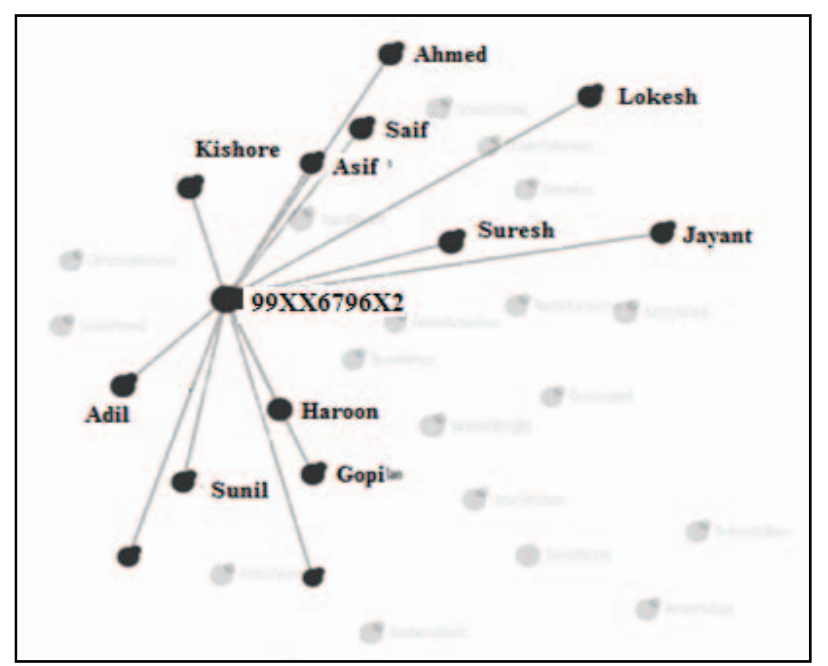

Fig. 7. Connection of Individual Suspects with Convicted Criminals

Now after analyzing the individual suspects association with the convicted criminals, police investigator can identify few names with whom the suspects are strongly associated. The strongness and weight factor of the association can be calculated based on various factors like number of calls made, number of messages exchanged etc. In our example case the suspect 99XX6796X5 is strongly associated (Figure 7) with the two convicted criminals: Jiten and Saif. The investigator has also found from the police record that these convicted criminals Jiten and Saif were also involved in the similar kind of crimes. Jiten and Saif are not directly linked to the crime we are investigating but they are in contact with someone who is our suspect (i.e. 99XX6796X5 in our case) and most likely to be a criminal who has committed the crime. We should focus our investigation on him.

\section{vi. Conclusion}

In our paper we have shown how in a few steps, we turned CDR call records analysis into one specific insight. In our criminal investigation we found that 99XX6796X5 is the likeliest suspect. In order to achieve the result, we simply used CDR Database with the power of graph analysis tools, which gave us very strong clue to crack the case. Crime investigation is one of the fields where graph analysis can be used, together with more traditional techniques, to discover insights in complex data. Investigating the case using archive CDR can give more insight, if it also takes input from social network profile of suspects and convicted criminals. As social network revels much information about the individual, it can be very useful for investigation. We are currently focusing on devising the techniques for analysis using CDR along with Social Network profile in efficient way.

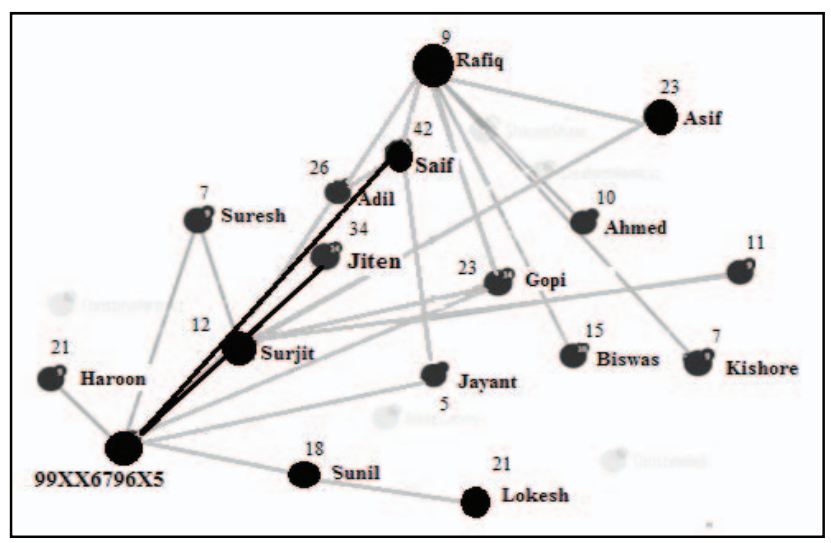

Fig. 6. Connection of Suspects with Convicted Criminals as per Weight Factors

\section{Acknowledgment}

I would like to thank MSRIT Management and my colleagues for their valuable suggestion, constant support and encouragement.

\section{References}

[1] F. Ozgul, C. Atzenbeck, A. Celik and Z. Erdem, "Incorporating data sources and methodologies for crime data mining," Intelligence and Security Informatics (ISI), 2011 IEEE International Conference on, Beijing, 2011, pp. 176-180.

[2] H. Chen, W. Chung, J. J. Xu, G. Wang, Y. Qin and M. Chau, "Crime data mining: a general framework and some examples," in Computer, vol. 37, no. 4, pp. 50-56, April 2004.

[3] Ilona Murynets and Roger Piqueras Jover. 2012. Crime scene investigation: SMS spam data analysis. In Proceedings of the 2012 ACM conference on Internet measurement conference (IMC '12). ACM, New York, NY, USA, 441-452.

[4] Lawrence McClendon and Natarajan Meghanathan, "Using Machine Learning To Analyze Crime Data", Machine Learning and Applications: An International Journal (MLAIJ) Vol.2, No.1, March 2015.

[5] R. Becker et al., "Clustering anonymized mobile call detail records to find usage groups", Proc. PURBA, pp. 1-8, Jun. 2011. 